\title{
Fortifications in the port area of Messina and Palermo between destruction, oblivion and debates on their restoration
} Carmen Genovese

Ministero dei Beni e delle Attività Culturali e del Turismo, Archivio di Stato, Palermo

mariacarmen.genovese@beniculturali.it

\begin{abstract}
The fortified structures constitute in Sicily a very important heritage, because of its shape and its geographic location.

Among many destructions of urban fortifications in the main cities of Sicily, the comparison between two of the most important cases, Messina and Palermo, can focus reason of the common destiny and factors who influenced the destruction of those monuments.

Messina boasted since 1680 the so-called "Cittadella", built by engineer Royal Military Carlos de Grunenbergh on sickle-shaped peninsula; survived the earthquake of 1908, unfortunately the old military structure was partly demolished to make way for modern port and industrial buildings.

Despite the partial demolition, the "Cittadella" is today a very significant monument, not only for its architectural and landscape value, but also because it is one of the few surviving examples of Messina as it was before the earthquake of 1908 , which, as is known, destroyed the city almost completely.

However, today, despite many studies carried out to date in an effort to restore and enhance the structure, it remains largely abandoned. Only the "Forte del Santissimo Salvatore", occupied by the Italian Navy, is in good condition and can be visited.

The "Castello a mare" in Palermo had Islamic origins; there were an important church and a monumental palace; in the twenties this fortified architecture was destroyed to build a new port and the superintendent Francesco Valenti managed to save , after a long and interesting debate, only part of the structures. The essay explores the events relating the destruction and conservation efforts of these two fortifications, with reflections on the political and speculative factors that influenced these events.
\end{abstract}

Keywords: Messina, Palermo, military structure, destruction, conservation.

\section{Introduzione}

In Sicilia il complesso e ricco sistema delle fortificazioni costiere costituisce un patrimonio monumentale di particolare importanza e consistenza sia per il suo essere isola, sia per la sua posizione baricentrica rispetto al bacino del Mediterraneo. Una delle peculiarità di molte architetture fortificate è quella di avere avuto una forte caratterizzazione simbolica e sociale non sempre positiva - pur avendo subito un ritardo nell'essere riconosciute come "monumenti", cioé docummenti architettonici della cultura difensiva ma anche costruttiva di un popolo.

Dapprima costruite a difesa delle città e dei cittadini, infatti, spesso le fortificazioni costiere castelli, fortilizi, mura - soprattutto in corrispondenza delle città, alla fine dell'Ottocento furono percepite non più come elemento di difesa, ma come un ostacolo al moderno sviluppo e come simbolo di oppressione politica. 
Seppur si debbano rilevare sporadici episodi di un precoce riconoscimento del loro valore storico ed architettonico - gli insediamenti fortificati spesso in Sicilia risalgono all'epoca della dominazione araba - in generale il loro destino, a partire dalla fine dell'Ottocento, fu accomunato da un progressivo abbandono, cessata la loro ragion d'essere, e spesso da deliberate azioni demolitive.

Dunque se è vero che la conservazione e la valorizzazione deve essere preceduta da un riconoscimento del monumento in quanto tale, nel caso delle fortificazioni questo processo culturale fu particolarmente difficile in quanto riguardava architetture nate per assolvere funzioni puramente utilitaristiche e, se vogliamo, di infausta memoria.

Per tali ragioni, nell'ambito della storia del restauro architettonico il tema delle fortificazioni e del loro destino tra abbandono, demolizioni e dibattiti, suggerisce alcune riflessioni che rimandano al più generale tema della necessità, per legittimare la conservazione delle architetture, di un pieno riconoscimento storico ed architettonico che sia condiviso a livello sociale, condizione che per le architetture fortificate non avvenne o avvenne molto tardi, quando buona parte di esse erano state già distrutte.

Tra la fine dell'Ottocento ed il primo Novecento si assiste in tutta la Sicilia - e non solo in questa regione - alla distruzione delle fortificazioni costiere, si pensi ai casi di Siracusa, Trapani e, naturalmente, Messina e Palermo.

Le fortificazioni che subirono maggiormente la furia devastatrice dell'uomo furono soprattutto a quelle poste all'interno delle città, certamente a causa dell'appetibilità dei vasti terreni in cui sorgevano. La speculazione edilizia e gli interessi commerciali infatti, mal celati da ragioni di modernità ed "igiene", furono i motori portanti di questa volontà distruttiva, come si costaterà anche nei due casi qui indagati.

Dall'analisi dei casi appare altrettanto chiaro che tali azioni furono spesso supportate da ideologie politiche e da un consenso popolare che faceva leva sulla volontà di cancellare quello che era diventato simbolo dell'oppressione borbonica, tanto più se esercitata dal regno sabaudo. In tale processo inntervennero anche le motivazioni legate all'unificazione nazionale, tese a cancellare ciò che era riconducibile alla difesa dei singoli stati e dunque alla frammentazione politica e culturale del nuovo territorio nazionale. Fu per tali ragioni che le fortificazioni - non solo cittadelle fortificate ma anche come molte porte urbane e cinte murarie - furono distrutte.

Le affinità riscontrate tra i due casi forse più emblematici della Sicilia, quelli degli insediamenti fortificati delle città di Messina e Palermo, consentono in questa sede di rafforzare i ragionamenti condotti. La Cittadella di Messina ed il Castello a mare di Palermo, infatti, non vengono qui analizzati nella loro lunga e significativa storia costruttiva, per la quale si rimanda ad altri contributi presenti anche nell'ambito di questo convegno, ma limitatamente alle vicende relative alla loro distruzione e/o conservazione, iniziate fondamentalmente alla fine dell'Ottocento.

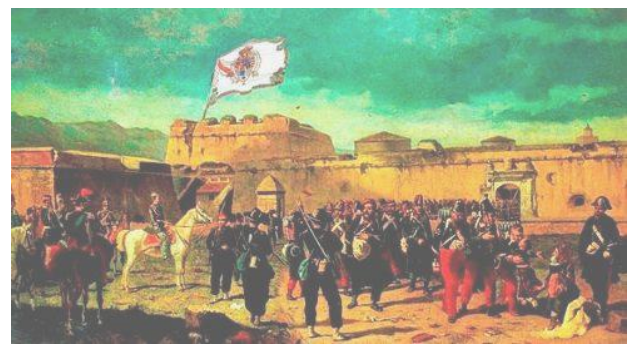

Fig. 1- G. Panebianco, La resa della Cittadella di Messina, 1892.

\subsection{Note sulla demolizione della Real Cittadella di Messina}

La questione della cosiddetta Cittadella di Messina è tornata solo negli ultimi anni ad essere dibattuta. In una città in cui le preesistenze architettoniche antecedenti il disastroso terremoto del 1908 sono rarissime, i resti della Cittadella, che invece resistettero a quel terribile terremoto, situati in un'area centralissima ed adiacente il porto, costituiscono un'evidente ferita aperta di abbandono e 
degrado, che attende ancora di essere restaurata e valorizzata.

La Real Cittadella era un forte a pianta stellare progettato dall'ingegnere Carlos de Grunenbergh alla fine del XVII secolo, non senza elementi di pregio architettonico - come le grandi porte di accesso scolpite, in piccola parte superstiti posta nel porto della città. Esso è caratterizzato da un allungamento di terra a forma di falce in cui esisteva già il forte del SS.Salvatore, che ancor oggi in buone condizioni in quanto sede della base della Marina Militare dal 1910 circa costiutisce una rara testimonianza della città cinquecentesca.

La Cittadella fu teatro di molti tra i principali avvenimenti nella città (Berdar, La Fauci, Riccobono, 1988); qui ci basti solo ricordare che, in occasione dei moti del 1848, dal suo interno fu dato ordine di bombare la città stessa per neutralizzare i rivoltosi; in seguito fu baluardo a difesa del Regno dai moti garibaldini del 1860 (fig. 1). La memoria di tali fatti incise sull'identificazione popolare post-unitaria della Cittadella come strumento di oppressione e quindi contribuì a decretarne l'abbattimento; «il popolo si duole di non veder demolire quegli inutili fortilizi che stanno sul collo della Città e del tutto sparire quelle orribili casematte di nefanda e dolorosa memoria», queste erano mediamente le considerazioni di chi, già nel 1861, auspicava un utilizzo diverso dell'area adiacente al porto (Berdar, La Fauci, Riccobono, 1988).

Negli anni Ottanta dell'Ottocento una politica di riarmo portò all'aggiornamento delle dotazioni difensive, con la modifica delle fortificazioni del porto di Messina e la costruzione di svariati forti a tutela dello Stretto, sia lungo le coste siciliane sia lungo quelle calabresi; questo complesso sistema difensivo "diffuso" costituisce oggi un patrimonio tanto significativo dal punto di vista architettonico e paesaggistico quanto - a parte rare eccezioni - ancora da valorizzare. «Nella seconda parte del XIX secolo in Italia si pone il problema della difesa dei territori e dei confini del nuovo Stato unitario (...) In questo panorama viene data molta importanza al ruolo militare della città di Messina, la più importante e nevralgica delle città dello Stretto» (Caruso, Lo Curzio, 2006).

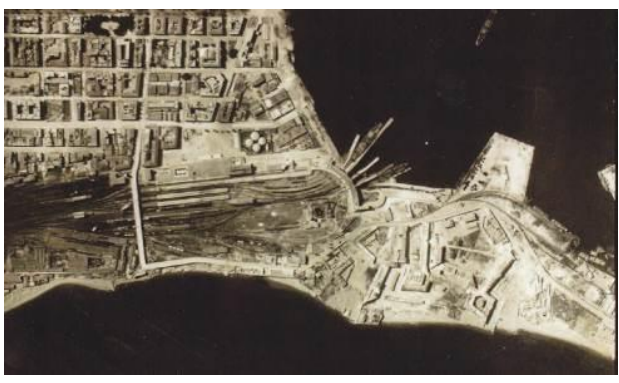

Fig. 2- Aerofoto del porto di Messina successiva alla realizzazione della stazione ferroviaria. Si noti il taglio della fortificazione a pianta stellare.

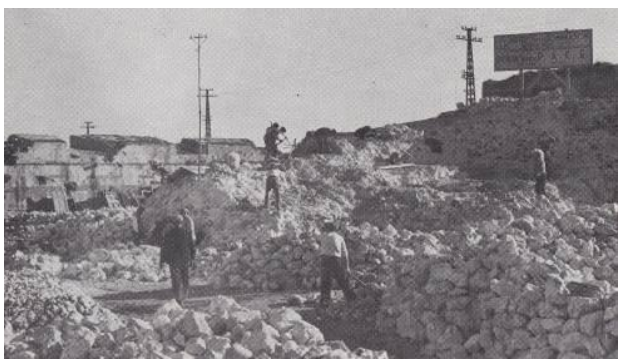

Fig. 3- Demolizione delle mura fortificate della Cittadella di Messina, 1939 ca. (Berdar, La Fauci, Riccobono, 1988).

Riparata dopo i moti ottocenteschi, col sisma del 1908 la Cittadella non subì molti danni, tanto che alcuni vasti ambienti voltati, rimasti pressoché indenni, furono adibiti a deposito di materiali giunti in città per far fronte all'emergenza post-sisma.

È singolare peraltro che nessuno, neppure il Soprintendente ai Monumenti della Sicilia Francesco Valenti, rilevò il valore architettonico e storico della Cittadella tentando di salvarla e restaurarla. La cosa stupisce perché Valenti fu protagonista del restauro e della ricostruzione dei monumenti messinesi dopo il sisma (Genovese 2010) e dimostrò, come si vedrà per il Castello a Mare di Palermo trattato in seguito, di saper riconoscere, in anticipo rispetto a quei tempi, il valore delle fortificazioni urbane.

Nessuno difese invece la Cittadella, danneggiata nei bombardamenti della seconda guerra mondiale, ma soprattutto via via smantellata in 
nome di una moderna sistemazione della stazione e del porto, nel primo Novecento (fig. 2-3), e per far spazio a disparate nuove attività e destinazioni d'uso che man mano venivano più o meno concesse dal Comune: officine, discariche, allevamenti, baracche abitative, perfino un inceneritore. Nonostante ciò, parzialmente sepolte dei detriti restano oggi parti significative dell'antica fortificazione stellare, di cui ormai quasi si ignorava l'esistenza.

Altre parti della Cittadella vennero demoliti per dare spazio alla nuova urbanizzazione, come nel caso del Forte Din Blasco, abbattuto per consentire il prolungamento della via Cannizzaro (fig. 4).

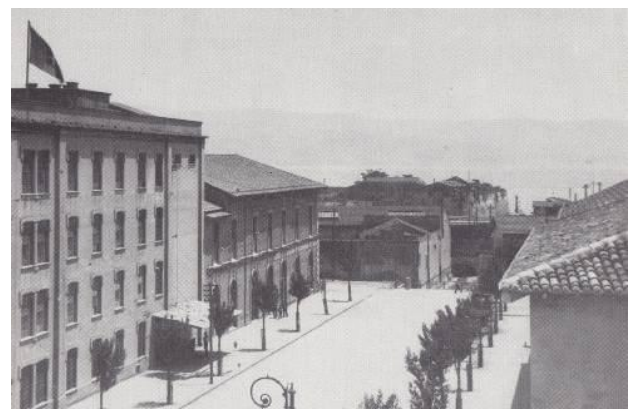

Fig. 4- Messina, via Cannizzaro intorno al 1930 e, in fondo, il Forte Don Blasco (Berdar, La Fauci, Riccobono, 1988).

Tra le poche azioni che denotano un riconoscimento del valore moumentale della Cittadella, nel Novecento non si effettuarono interventi diretti di conservazione, ma di smembramento, come lo smontaggio, nel 1961, della Porta Grazia, per essere innestata in un lacerto lapideo in una piazza cittadina: operazione, di per sè, rinunciataria di qualsiasi proposito di recupero dei resti della fortificazione messinese (fig. 5).

Recentissimamente il dibattito sulla Cittadella sembra essersi nuovamente rinvigorito, nella speranza che a ciò possano seguire reali interventi di restauro e valorizzazione. In tema di rifunzionalizzazione e valorizzazione del patrimonio fortificato dello Stretto, si segnala come recente esempio positivo il passaggio dallo Stato al Comune di Campo Calabro del Fortino ottocentesco Poggio-Pignatelli, fino ad allora in disuso; l'accordo è stato possibile a fronte della presentazione di un adeguato programma di valorizzazione. L'operazione ha visto partecipi le Amministrazioni competenti, dall'Agenzia del Demanio al Ministero per i Beni e le Attività Culturali, in particolare la Direzione Regionale per i Beni Culturali e Paesaggistici della Calabria, grazie all'applicazione della recente normativa sul federalismo demaniale per i beni di interesse storico-artistico.

L'auspicio è che anche per la Cittadella possa avverarsi una efficace collaborazione tra Amministrazioni competenti finalizzata alla riappropriazione dei resti della fortificazione da parte della città di Messina e dall'individuazione di un adeguato programma di "conservazione integrata".

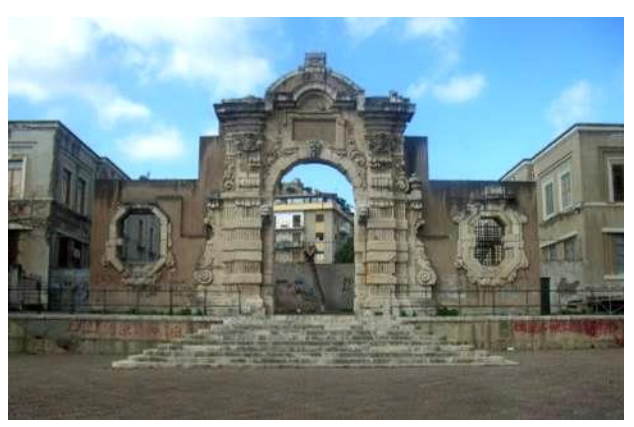

Fig. 5- Porta Grazia nella sua collocazione attuale, dopo essere stata smontata dalla Cittadella e sistemata in una piazza della città.

\subsection{Note sulla demolizione del Castello a Mare di Palermo}

Il Forte Castellammare, o Castello a mare sorgeva, su preesistenze di origine araba, accanto al porto di Palermo, detto "la Cala". Nel Cinquecento fu sede dei Viceré e del Tribunale della Santa Inquisizione.

Anche il Forte palermitano fu teatro di scontri durante i moti del 1848, subendo le prime distruzioni, e divenne poi presidio militare contro eventuali rivolte. Negli anni seguenti all'interno delle sue carceri vi furono molti prigionieri politici e la sua chiesa veniva ricordata per aver ospitato prima della morte alcuni eroi antiborbonici. Durante l'assedio garibaldini, nel 1860, analogamente a quanto 
accadeva a Messina, dal Forte palermitano si aprì il fuoco verso la città.

Dopo tali eventi, perfettamente in linea con gli orientamenti dell'urbanistica in Europa, è comprensibile che le prospettive pianificatorie della città - si pensi al Piano Giarrusso del 1885 - prevedessero l'abbattimento del Forte, insieme a tratti di mura ed a porte urbiche, operazioni all'epoca giustificate tecnicamente dalla necessità di ventilare i malsani quartieri del centro, dunque ancora una volta per motivi di "igiene".

Già alla fine dell'Ottocento si registrano propositi di demolizione del Forte tanto che l'allora Direttore dell'Ufficio regionale dei monumenti, Giuseppe Patricolo, nel 1899, concedeva parere favorevole alla demolizione: «Di fronte alla ragione edilizia e igienica, specialmente, (...) tenuto conto che la importanza degli avanzi (...) non è poi tale da impedirne la demolizione, così credo che si possa senz'altro accordare al Ministero della Guerra il chiesto nulla osta» (Di Stefano C.A., Lo Iacono G., 2012).

Evidentemente i tempi non erano ancora maturi perchè si tutelasse un'architettura come il Castello a mare e neppure Patricolo, che pur si distinse per un'intensa attività di restauro dei principali monumenti palermitani (Tomaselli, 1994), non ne seppe cogliere il valore. Dopo vari tentativi dunque anche a Palermo le ragioni dello sviluppo urbano inducevano, negli anni Venti, all'ampliamento del porto della città. Il progetto prevedeva, a tal fine, la completa distruzione del Forte Castellammare, seppur fondata su antiche preesistenze e dotata di elementi architettonici e decorativi di pregio.

L'operazione sollecitò anche gli interessi di un banchiere americano, e che fu costituita la Società Anonima Italiana Mac Arthur appositamente per eseguire i lavori per il nuovo porto.

La ditta incaricata era già in procinto di demolire il Forte, con l'approvazione del Ministero dei Lavori Pubblici, quando si opponeva un Comitato cittadino per la conservazione dei monumenti, promosso da intellettuali tra cui l'architetto Ernesto Basile.
Il già citato Soprintendente ai Monumenti della Sicilia, l'ingegnere Francesco Valenti, e quello alle Gallerie, l'archeologo Ettore Gabrici, si fecero portavoce di tali proteste, chiedendo che almeno parte delle antiche strutture fossero il più possibile conservate.

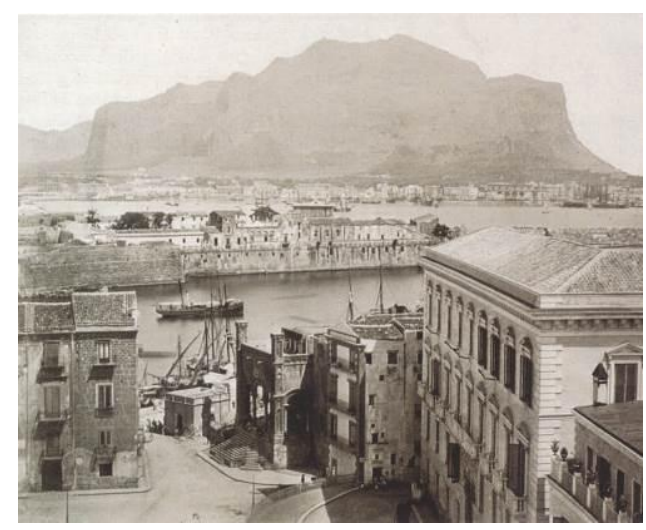

Fig. 6- La "Cala" di Palermo intorno al 1870. Si noti il fronte del palazzo cinquecentesco del Castello a mare (foto di G. Incorpora).

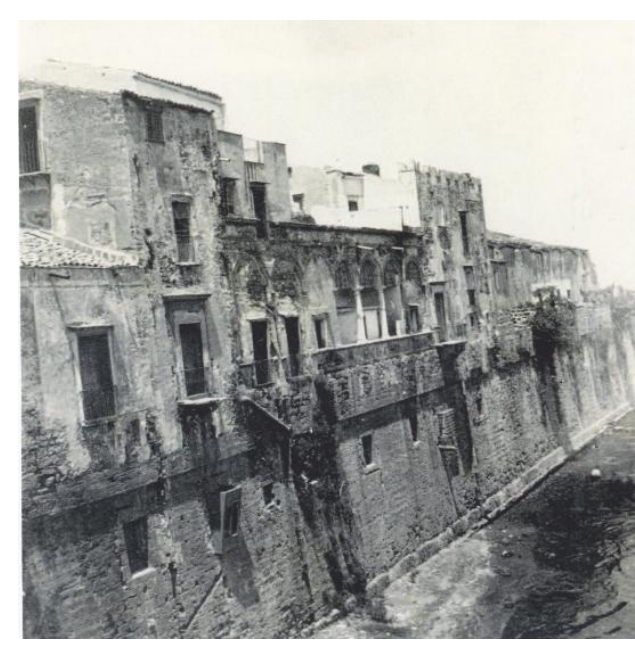

Fig. 7- Il palazzo con la loggia cinquecentesca prima delle demolizioni (Fondo Valenti).

Il partecipatissimo dibattito che si innescò, ben documentato nelle pagine dei giornali locali, nell'archivio storico della Soprintendenza e in quello privato di Valenti, conservato presso l'Archivio comunale palermitano, costituisce una pagina significativa nella storia del restauro in Sicilia e probabilmente, per l'avanzamento 
delle posizioni mostrate a difesa della conservazione di questa fortificazione, anche nel quadro del restauro nazionale.

Se Valenti non aveva in nessun modo riconosciuto il valore della Cittadella di Messina - probabilmente sopraffatto da più gravi emergenze postegli dalla città distrutta dal terremoto - a Palermo, sua città natale, egli assume una posizione avanzata rispetto ai coevi a favore del riconoscimento e della conservazione del Castello a mare, che certamente meglio conosceva: «sono venute alla luce le murature antiche del maschio arabo normanno del Castello medesimo, e sono apparsi particolari interessantissimi della costruzione militare dei secoli XI e XII (...).

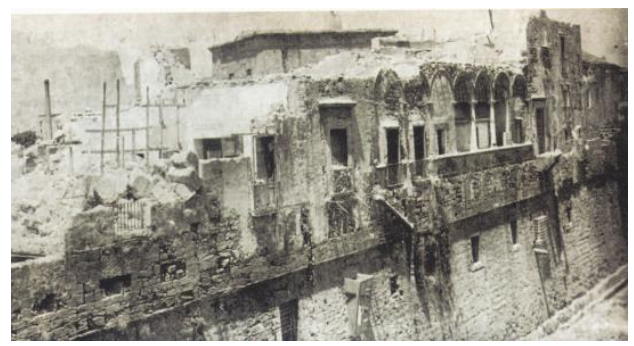

Fig. 8- Il palazzo con la loggia cinquecentesca durante le demolizioni, eseguite negli anni Venti del Novecento (La Duca, 1980).
Tali rinvenimenti hanno un importante interesse per l'arte e per la storia». In tali valutazioni gli fu probabilmente d'aiuto l'esperienza del lungo restauro del Palazzo Reale palermitano, ed è apprezzabile, nel 1928, la sua volontà di conservare i resti del Forte «per raggiungere lo scopo di far conoscere agli studiosi questo caposaldo della difesa della città nei sec. X a XII e per imporne la conservazione tanto contrastata anche dalle autorità del passato Regime» (Fondo Valenti).

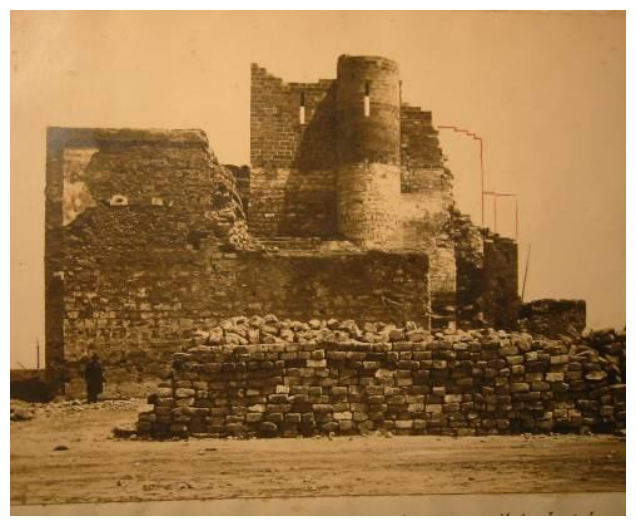

Fig. 9- Veduta ovest dei resti del Forte con una torre circolare, rinvenuta durante le demolizioni. Le linee a destra, tratteggiate da Valenti, indicano in didascalia «la parte crollata da rialzare col materiale caduto» (Fondo Valenti).

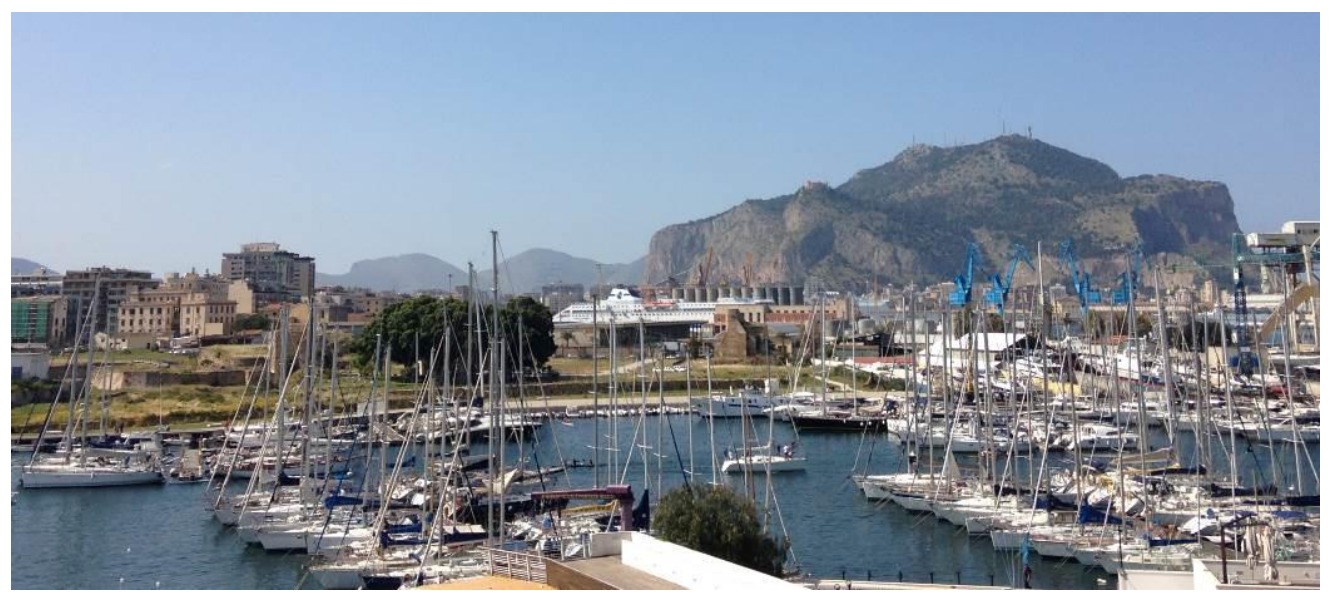

Fig. 10- Vista della "Cala" di Palermo oggi. Si intravedono i resti basamentali del palazzo cinquecentesco e le poche emergenze del Castello a mare salvate dal Soprintendente Valenti. 
Purtroppo - cosa comprensibile considerata l'epoca - il riconoscimento del Forte come monumento non fu posizione largamente condivisa, ed il popolo salutò con giubilo la venuta della società americana e la pomposa inaugurazione dei lavori, in occasione della quale il Re Vittorio Emanuele III in persona fece brillare nel 1922 le prime mine di inizio dei lavori di demolizione. Sono significative le parole del Sindaco in quella occasione: «come il progresso umano non edifica senza demolire (..), inizia pure la distruzione di quanto si oppone al futuro (...) che fu il nostro forte di Castellammare. Per la verità esso non vanta glorie né di storia né di arte; servì verso Palermo come strumento meno di difesa, che di offesa e di oppressione tirannica (...) e ora finalmente Palermo saluta la scomparsa dell suo vecchio castello senza più alcun rancore, ma anche senza eccessivo rimpianto» (Di Stefano C.A., Lo Iacono G., 2012).

Poco poté fare Valenti: proprio mentre richiedeva al Consiglio Superiore delle Antichità e Belle Arti un'ispezione che mai giunse ed inviava solleciti alle autorità competenti - di cui resta un corposo carteggio - fu demolita in tutta fretta gran parte dell'antico forte, tra cui la chiesa ed il palazzo cinquecentesco sede dei Viceré, di caratter monumentale con portico rinascimentale rilevato da Valenti come elemento di pregio (figg. 6-9).

$\mathrm{Si}$ riuscì a conservare ben poco, come il cosiddetto "maschio arabo normanno" rinvenuto nel corso delle demolizioni della struttura, a pianta circolare, ed il corpo d'ingresso. Le proteste di Valenti presso l'impresa ed il Comune ebbero quindi esito parziale, mentre l'impresa procedeva comunque alle demolizioni per tutelare i propri interessi economici, «adducendo la scusa della disoccupazione operaia». La Soprintendenza redasse anche un progetto di consolidamento e conservazione, che fu possibile eseguire sui pochi resti superstiti dalle demolizioni; nel frattempo si accanivano su di essi con atti vandalici anche ignoti, probabilmente in accordo con la ditta demolitrice o comunque con chi era contrario alla conservazione. In una lettera al Ministero,
Valenti lamentava l'inefficacia della sua azione, «mentre si demolivano vandalicamente altri resti importanti dei sec. XV e XVI per ottenere una grande spianata dove ancora non si sa quale disposizione dovranno avere gli edifici dipendenti dalla nuova sistemazione del porto».

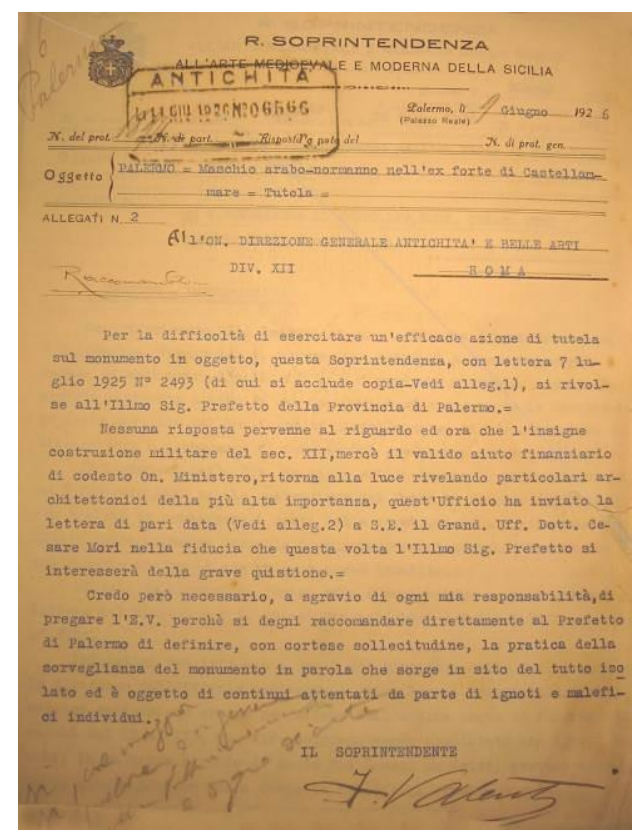

Fig. 11- Nota di Valenti alla Direzione Generale Antichità e Belle Arti sulla questione del Castello a mare di Palermo (Archivio Soprintendenza).

In queste parole si ritrovano le ragioni portate avanti da Valenti anche nell'ambito di molti altri interventi, in cui, proprio dal primo Novecento in poi molti brani di intere città soccombevano in nome dell'urbanizzazione; dietro i motivi di "igiene" si celavano motivazioni ideologiche e, ancor più spesso, interessi economici e speculativi, in cui ebbero la peggio soprattutto quegli edifici ritenuti ormai inutili, come le fortificazioni urbane. La pietra fatta pazientemente recupere dalla demolizione del cosiddetto "ex forte Castellammare" fu poi usata dalla stessa Soprintendenza per eseguire restauri e consolidamenti dei monumenti palermitani, come per il prospetto sud della Cattedrale, nel 1924 (Genovese 2010). 
A partire dal 1988 i pochi resti del Castello a mare di Palermo ebbero sorte migliore di quelli della fortezza messinese e, restaurati e valorizzati dalla Soprintendenza competente, oggi sono visitabili e fanno parte di un Parco archeologico che accoglie peraltro numerosi eventi culturali (fig. 10).

\section{Conclusioni}

Il destino di abbandono e distruzione che accomunò anche in Sicilia molte architeture fortificate tra Otto e Novecento costituisce non solo una storia da ricordare per di riscoprire architetture ancor oggi dimenticate, ma è ancor oggi il paradigma di come ideologie politiche, economiche e speculative possano in ogni epoca influenzare la percezione colletitva dei valori di cui un'architettura è depositaria in quanto monumento e quindi decretarne la distruzione.

Seppur il moderno processo di riconoscimento di un monumento sia ben diverso ed avanzato rispetto a quello di un secolo fa, è innegabile che gli interessi economici mettono ancor oggi in pericolo una parte del patrimonio che per vari fattori non ha ancora avuto pienno riconoscimento dalla nostra società - si pensi ad esempio all'architettura dei primi del Novecento o alla cosiddetta archeologia industruale.

D'altronde ancora molto rimane da fare, come nel caso di Messina, per recuperare una parte della nostra storia, quella delle fortificazioni, che troppo spesso è stata sottovalutata se non ignorata nelle sue valenze storiche ed architettoniche.

\section{References}

Archivio Comunale di Palermo, Fondo Valenti, Forte Castellammare di Palermo.

Archivio Storico della Soprintendenza BB. CC. AA., Forte Castellammare di Palermo.

Berdar A., La Fauci C., Riccobono F. (1988). La Real Cittadella di Messina. Ed. Dott. Antonio Sfameni. Messina.

Caruso V., Lo Curzio M. (2006). La fortificazione permanente dello Stretto di Messina. Storia, conservazione e restauro di un patrimonio architettonico e ambientale. EDAS. Messina.

Di Stefano C.A., Lo Iacono G. (2012). Il Castello a mare di Palermo. Cronostoria della demolizione di un monumento. Edit Opera. Palermo.

Genovese C. (2010). Francesco Valenti. Restauro dei monumenti nella Sicilia del primo Novecento. Edizioni Scientifiche Italiane. Napoli.

La Duca R. (1980). Il castello a mare di Palermo. EPOS. Palermo.

Tomaselli F. (1994), Il ritorno dei Normanni, Gangemi Editore, Roma. 